Article

\title{
On Fractional Symmetric Hahn Calculus
}

\author{
Nichaphat Patanarapeelert ${ }^{1}$ and Thanin Sitthiwirattham ${ }^{2, * \mathbb{D}}$ \\ 1 Department of Mathematics, Faculty of Applied Science, King Mongkut's University of Technology \\ North Bangkok, Bangkok 10800, Thailand; nichaphat.p@sci.kmutnb.ac.th \\ 2 Mathematics Department, Faculty of Science and Technology, Suan Dusit University, \\ Bangkok 10300, Thailand \\ * Correspondence: thanin_sit@dusit.ac.th
}

Received: 14 August 2019; Accepted: 17 September 2019; Published: 20 September 2019

Abstract: In this paper, we study fractional symmetric Hahn difference calculus. The new idea of the symmetric Hahn difference operator, the fractional symmetric Hahn integral, and the fractional symmetric Hahn operators of Riemann-Liouville and Caputo types are presented. In addition, we formulate some fundamental properties based on these fractional symmetric Hahn operators.

Keywords: fractional symmetric Hahn integral; fractional symmetric Hahn difference operator

JEL Classification: 39A10; 39A13; 39A70

\section{Introduction}

The Hahn difference operator, one type of quantum difference operator, has been studied by many reseachers. It is used to construct families of orthogonal polynomials and to study certain approximation problems (see [1-3]).

Hahn [4] is the first researcher who introduced the Hahn difference operator $D_{q, \omega}$ based on the forward difference operator and the Jackson $q$-difference operator where

$$
D_{q, \omega} f(t):=\frac{f(q t+\omega)-f(t)}{t(q-1)+\omega}, t \neq \omega_{0}:=\frac{\omega}{1-q} .
$$

Later, the right inverse of Hahn's operator and its properties were presented (see $[5,6])$. There are other works related to the Hahn difference operator such as the study of Hahn quantum variational calculus [7-9], and the existence and uniqueness results for the initial value problems [10-12] and boundary value problems $[13,14]$.

Recently, Brikshavana and Sitthiwirattham [15] introduced fractional Hahn difference operators. The boundary value problems for fractional Hahn difference equations were subsequently studied by many researchers (see [16-19]).

In 2013, Artur et al. [20] introduced the symmetric Hahn difference operator $\tilde{D}_{q, \omega}$ as

$$
\tilde{D}_{q, \omega} f(t):=\frac{f(q t+\omega)-f\left(q^{-1}(t-\omega)\right)}{\left(q-q^{-1}\right) t+\left(1+q^{-1}\right) \omega} \text { for } t \neq \omega_{0} .
$$

However, we observe from the literature that fractional symmetric Hahn difference calculus has not been studied. In order to give a rigorous analysis of symmetric Hahn calculus, this paper is devoted to presenting the new concepts of the symmetric Hahn difference operator, the fractional symmetric Hahn integral, and the fractional symmetric Hahn difference operators of the Riemann-Liouville and Caputo types. Particularly, the results from this study can be used as a tool in some applications 
such as approximation problems, and initial and boundary value problems associated with symmetric Hahn operators. We first introduce some basic definitions and properties of Hahn's difference operators in Section 2. In Section 3, we present the fractional symmetric Hahn integral and its properties. Finally, we propose the fractional symmetric Hahn difference operators of the Riemann-Liouville and Caputo types and their properties in Sections 4 and 5, respectively.

\section{Preliminary Definitions and Properties}

In order to study the fractional symmetric Hahn difference calculus, we first introduce some notations, definitions, lemmas as follows. (see [4-8,20,21]).

For $0<q<1, \omega>0, \omega_{0}=\frac{\omega}{1-q}$, we define

$$
\begin{aligned}
& \widetilde{[k]_{q}}:= \begin{cases}\frac{1-q^{2 k}}{1-q^{2}}=[k]_{q^{2}}, & k \in \mathbb{N} \\
1, & k=0,\end{cases} \\
& \widetilde{[k]_{q}} !:= \begin{cases}\left.\widetilde{[k}]_{q}[\widetilde{k-1}]_{q} \cdots \widetilde{11}\right]_{q}=\prod_{i=1}^{k} \frac{1-q^{2 i}}{1-q^{2}}, & k \in \mathbb{N} \\
1, & k=0 .\end{cases}
\end{aligned}
$$

The $q, \omega$-forward jump operator is defined by

$$
\sigma_{q, \omega}^{k}(t):=q^{k} t+\omega[k]_{q},
$$

and the $q, \omega$-backward jump operator is defined by

$$
\rho_{q, \omega}^{k}(t):=\frac{t-\omega[k]_{q}}{q^{k}}
$$

where $k \in \mathbb{N}$.

Letting $n \in \mathbb{N}_{0}:=\{0,1,2, \ldots\}, a, b \in \mathbb{R}$, we define the power functions as follows:

- The $q$-analogue of the power function

$$
(a-b) \frac{0}{q}:=1, \quad(a-b) \frac{n}{q}:=\prod_{i=0}^{n-1}\left(a-b q^{i}\right),
$$

- $\quad$ The $q$-symmetric analogue of the power function

$$
(\widetilde{a-b})_{q}^{\underline{0}}:=1, \quad(\widetilde{a-b})_{q}^{\frac{n}{q}}:=\prod_{i=0}^{n-1}\left(a-b q^{2 i+1}\right),
$$

- The $q, \omega$-symmetric analogue of the power function

$$
(\widetilde{a-b})_{q, \omega}^{\frac{0}{2}}:=1, \quad(\widetilde{a-b})_{q, \omega}^{\frac{n}{2}}:=\prod_{i=0}^{n-1}\left[a-\sigma_{q, \omega}^{2 i+1}(b)\right] .
$$

In general, for $\alpha \in \mathbb{R}$, we have

$$
\begin{gathered}
(a-b) \frac{\alpha}{q}=a^{\alpha} \prod_{i=0}^{\infty} \frac{1-\left(\frac{b}{a}\right) q^{i}}{1-\left(\frac{b}{a}\right) q^{\alpha+i}}, a \neq 0, \\
(\widetilde{a-b})_{q}^{\underline{\alpha}}=a^{\alpha} \prod_{i=0}^{\infty} \frac{1-\left(\frac{b}{a}\right) q^{2 i+1}}{1-\left(\frac{b}{a}\right) q^{2(\alpha+i)+1}}, a \neq 0 .
\end{gathered}
$$


Since

$$
\begin{aligned}
(\widetilde{a-b})_{q, \omega}^{\underline{n}} & =\prod_{i=0}^{n-1}\left[a-\sigma_{q, \omega}^{2 i+1}(b)\right]=\prod_{i=0}^{n-1}\left[\left(a-\omega_{0}\right)-\left(b-\omega_{0}\right) q^{2 i+1}\right] \\
& =\left(\left(a-\omega_{0}\right)-\left(b-\omega_{0}\right)\right)_{q}^{\underline{n}} \\
& =\left(a-\omega_{0}\right)^{n} \prod_{i=0}^{n-1}\left[1-\left(\frac{b-\omega_{0}}{a-\omega_{0}}\right) q^{2 i+1}\right] \cdot \frac{\prod_{i=n}^{\infty}\left[1-\left(\frac{b-\omega_{0}}{a-\omega_{0}}\right) q^{2 i+1}\right]}{\prod_{i=n}^{\infty}\left[1-\left(\frac{b-\omega_{0}}{a-\omega_{0}}\right) q^{2 i+1}\right]} \\
& =\left(a-\omega_{0}\right)^{n} \prod_{i=0}^{\infty} \frac{1-\left(\frac{b-\omega_{0}}{a-\omega_{0}}\right) q^{2 i+1}}{1-\left(\frac{b-\omega_{0}}{a-\omega_{0}}\right) q^{2(n+i)+1}}
\end{aligned}
$$

so, we obtain

$$
(\widetilde{a-b}))^{\frac{\alpha}{q}, \omega}=\left(\left(a-\omega_{0}\right)-\left(b-\omega_{0}\right)\right)_{q}^{\underline{\alpha}}=\left(a-\omega_{0}\right)^{\alpha} \prod_{i=0}^{\infty} \frac{1-\left(\frac{b-\omega_{0}}{a-\omega_{0}}\right) q^{2 i+1}}{1-\left(\frac{b-\omega_{0}}{a-\omega_{0}}\right) q^{2(\alpha+i)+1}}, a \neq \omega_{0} .
$$

In particular, if $a \neq b=0$, we have $a_{q}^{\alpha}=\widetilde{a}_{q}^{\alpha}=a^{\alpha}$. If $a \neq b=\omega_{0}$, we have $\left(\widetilde{a-\omega_{0}}\right)_{q, \omega}^{\underline{\alpha}}=\left(a-\omega_{0}\right)^{\alpha}$. Furthermore, if $a=b=0$, we define $\left.(0) \frac{\alpha}{q}=\widetilde{(0)_{q}} \frac{\alpha}{q}=\widetilde{(0}\right)_{q, \omega}^{\underline{\alpha}}:=0$ for $\alpha>0$.

Next, we define $q$-symmetric gamma and $q$-symmetric beta functions as

$$
\begin{aligned}
\tilde{\Gamma}_{q}(x) & := \begin{cases}\frac{\left(1-q^{2}\right) \frac{x-1}{q}}{\left(1-q^{2}\right)^{x-1}}=\frac{(\widetilde{1-q})^{\frac{x-1}{q}}}{\left(1-q^{2}\right)^{x-1}}, & x \in \mathbb{R} \backslash\{0,-1,-2, \ldots\} \\
{[x-1]_{q} !,} & x \in \mathbb{N}\end{cases} \\
\tilde{B}_{q}(x, y) & :=\frac{\tilde{\Gamma}_{q}(x) \tilde{\Gamma}_{q}(y)}{\tilde{\Gamma}_{q}(x+y)},
\end{aligned}
$$

respectively.

Lemma 1. For $m, n \in \mathbb{N}_{0}$ and $\alpha \in \mathbb{R}$,

(a) $\quad\left(x-\widetilde{\sigma_{q, \omega}^{n}}(x)\right)_{q, \omega}^{\underline{\alpha}}=\left(x-\omega_{0}\right)^{k}\left(\widetilde{1-q^{n}}\right)_{q^{\prime}}^{\underline{\alpha}}$

(b) $\left(\sigma_{q, \omega}^{m}(x) \widetilde{-} \sigma_{q, \omega}^{n}(x)\right)_{q, \omega}^{\underline{\alpha}}=q^{m \alpha}\left(x-\omega_{0}\right)^{\alpha}\left(1-\widetilde{q^{n}}-m\right)_{q}^{\frac{\alpha}{q}}$.

Proof. For $m, n \in \mathbb{N}_{0}$ and $\alpha \in \mathbb{R}$, we have

$$
\begin{aligned}
\left(x-\widetilde{\sigma_{q, \omega}^{n}}(x)\right)_{q, \omega}^{\underline{\alpha}} & =\left(\left(x-\omega_{0}\right)-\widetilde{\left.\left(\sigma_{q, \omega}^{n}(x)-\omega_{0}\right)\right)_{q}^{\underline{k}}}\right. \\
& =\left(x-\omega_{0}\right)^{\alpha} \prod_{i=0}^{\infty} \frac{1-\left(\frac{\sigma_{q, \omega}^{n}(x)-\omega_{0}}{x-\omega_{0}}\right) q^{2 i+1}}{1-\left(\frac{\sigma_{q, \omega}^{n}(x)-\omega_{0}}{x-\omega_{0}}\right) q^{2(i+\alpha)+1}} \\
& =\left(x-\omega_{0}\right)^{\alpha} \prod_{i=0}^{\infty} \frac{1-q^{n} q^{2 i+1}}{1-q^{n} q^{2(i+\alpha)+1}} \\
& =\left(x-\omega_{0}\right)^{\alpha}\left(1-q^{n}\right)_{q}^{\underline{\alpha}} .
\end{aligned}
$$


and

$$
\begin{aligned}
\left(\left(\sigma_{q, \omega}^{m}(x) \widetilde{)}-\sigma_{q, \omega}^{n}(x)\right)_{q, \omega}^{\underline{\alpha}}\right. & =\left(\left(\sigma_{q, \omega}^{m}(x)-\omega_{0}\right)-\left(\sigma_{q, \omega}^{n}(x)-\omega_{0}\right)\right)_{q}^{\underline{\alpha}} \\
& =\left(\left(\sigma_{q, \omega}^{m}(x)-\omega_{0}\right)^{\alpha} \prod_{i=0}^{\infty} \frac{1-\left(\frac{\sigma_{q, \omega}^{n}(x)-\omega_{0}}{\sigma_{q, \omega}^{m}(x)-\omega_{0}}\right) q^{2 i+1}}{1-\left(\frac{\sigma_{q, \omega}^{n}(x)-\omega_{0}}{\sigma_{q, \omega}^{m}(x)-\omega_{0}}\right) q^{2(i+\alpha)+1}}\right. \\
& =\left(q^{m}\left(x-\omega_{0}\right)\right)^{\alpha} \prod_{i=0}^{\infty} \frac{1-q^{n-m} q^{2 i+1}}{1-q^{n-m} q^{2(i+\alpha)+1}} \\
& =q^{m \alpha}\left(x-\omega_{0}\right)^{\alpha}\left(1-q^{n-m}\right)_{q}^{\underline{\alpha}} .
\end{aligned}
$$

So, Lemma 1 (a) and Lemma 1 (b) hold. The proof is complete.

Lemma 2. Let $t, s \in I_{q, \omega}^{T}:=\left\{q^{k} T+\omega[k]_{q}: k \in \mathbb{N}_{0}\right\} \cup\left\{\omega_{0}\right\}, T>\omega_{0}$. Then,

$$
(\widetilde{t-s})_{q, \omega}^{\underline{\alpha}}=0
$$

where $t \geq s$ and $\alpha \notin \mathbb{N}_{0}$.

Proof. Since $t, s \in I_{q, \omega}^{T}$, we have $t=\sigma_{q, \omega}^{m}(T), s=\sigma_{q, \omega}^{n}(T)$ where $m, n \in \mathbb{N}$. For $t \geq s$, we find that

$$
\begin{aligned}
\widetilde{(\tau-s)_{q, \omega}^{\underline{\alpha}}} & =\left(\sigma_{q, \omega}^{m}(T) \widetilde{-\sigma_{q, \omega}^{n}}(T)\right)_{q, \omega}^{\underline{\alpha}} \\
& =q^{m \alpha}\left(T-\omega_{0}\right)^{\alpha}\left(1-\widetilde{q^{n}-m}\right)_{q}^{\underline{\alpha}} \\
& =q^{\alpha m}\left(T-\omega_{0}\right)^{\alpha} \prod_{i=0}^{\infty}\left[\frac{1-q^{2 i+n-m+1}}{1-q^{2 i+n-m+1+2 \alpha}}\right]=0 .
\end{aligned}
$$

The proof is complete.

Definition 1 ([20]). For $q \in(0,1), \omega>0$, we let $f$ be the function defined on $I_{q, \omega}^{T} \subseteq \mathbb{R}$. The symmetric Hahn difference of $f$ is defined by

$$
\begin{aligned}
& \tilde{D}_{q, \omega} f(t):=\frac{f\left(\sigma_{q, \omega}(t)\right)-f\left(\rho_{q, \omega}(t)\right)}{\sigma_{q, \omega}(t)-\rho_{q, \omega}(t)} \quad t \in I_{q, \omega}^{T}-\left\{\omega_{0}\right\}, \\
& \tilde{D}_{q, \omega} f\left(\omega_{0}\right)=f^{\prime}\left(\omega_{0}\right) \text { where } f \text { is differentiable at } \omega_{0} .
\end{aligned}
$$

$\tilde{D}_{q, \omega} f$ is called $q, \omega$-symmetric derivative of $f$, and $f$ is $q, \omega$-symmetric differentiable on $I_{q, \omega}^{T}$.

From the above definition, we note that

$$
\tilde{D}_{q, \omega}^{0} f(x)=f(x) \text { and } \tilde{D}_{q, \omega}^{N} f(x)=\tilde{D}_{q, \omega} \tilde{D}_{q, \omega}^{N-1} f(x) \text { where } N \in \mathbb{N} .
$$

Lemma 3 ([20]). Properties of symmetric Hahn difference operators If $f$ and $g$ are $q, \omega$-symmetric differentiable on $I_{q, \omega}^{T}$. Then

(a) $\tilde{D}_{q, \omega}[f(t)+g(t)]=\tilde{D}_{q, \omega} f(t)+\tilde{D}_{q, \omega} g(t)$,

(b) $\quad \tilde{D}_{q, \omega}[f(t) g(t)]=f\left(\rho_{q, \omega}(t)\right) \tilde{D}_{q, \omega} g(t)+g\left(\sigma_{q, \omega}(t)\right) \tilde{D}_{q, \omega} f(t)$,

(c) $\tilde{D}_{q, \omega}\left[\frac{f(t)}{g(t)}\right]=\frac{g\left(\rho_{q, \omega}(t)\right) \tilde{D}_{q, \omega} f(t)-f\left(\rho_{q, \omega}(t)\right) \tilde{D}_{q, \omega} g(t)}{g\left(\rho_{q, \omega}(t)\right) g\left(\sigma_{q, \omega}(t)\right)}$ for $g\left(\rho_{q, \omega}(t)\right) g\left(\sigma_{q, \omega}(t)\right) \neq 0$,

(d) $\tilde{D}_{q, \omega}[C]=0$ where $C$ is constant. 
Lemma 4. Let $0<q<1, \omega>0, t \in I_{q, \omega}^{T}$, and $\alpha, \beta \in \mathbb{R}$. Then,
(a) $\left.\tilde{D}_{q, \omega}(\widetilde{t-\beta})_{q, \omega}^{\underline{\alpha}}=\widetilde{[\alpha]_{q}}\left(\rho_{q, \omega} \widetilde{(t)}-\beta\right)\right)_{q, \omega}^{\frac{\alpha-1}{}}$,
(b) $\tilde{D}_{q, \omega}(\widetilde{\beta-t})_{q, \omega}^{\underline{\alpha}}=-\widetilde{[\alpha]_{q}}\left(\beta-\widetilde{\sigma_{q, \omega}}(t)\right)_{q, \omega}^{\frac{\alpha-1}{q}}$.

Proof. By Lemma 1 and Definition 1, we find that

$$
\begin{aligned}
& \tilde{D}_{q, \omega}(t-\beta) \frac{\alpha}{q, \omega}=\tilde{D}_{q, \omega}\left[\left(t-\omega_{0}\right)^{\alpha} \prod_{i=0}^{\infty}\left(\frac{1-\left(\frac{\beta-\omega_{0}}{t-\omega_{0}}\right) q^{2 i+1}}{1-\left(\frac{\beta-\omega_{0}}{t-\omega_{0}}\right) q^{2(i+\alpha)+1}}\right)\right] \\
& =\frac{1}{\sigma_{q, \omega}(t)-\rho_{q, \omega}(t)}\left\{\left(\sigma_{q, \omega}(t)-\omega_{0}\right)^{\alpha} \prod_{i=0}^{\infty}\left(\frac{1-\left(\frac{\beta-\omega_{0}}{\sigma_{q, \omega}(t)-\omega_{0}}\right) q^{2 i+1}}{1-\frac{\beta-\omega_{0}}{\sigma_{q, \omega}(t)-\omega_{0}} q^{2(i+\alpha)+1}}\right)\right. \\
& \left.-\left(\rho_{q, \omega}(t)-\omega_{0}\right)^{\alpha} \prod_{i=0}^{\infty}\left(\frac{1-\left(\frac{\beta-\omega_{0}}{\rho_{q, \omega}(t)-\omega_{0}}\right) q^{2 i+1}}{1-\left(\frac{\beta-\omega_{0}}{\rho_{q, \omega}(t)-\omega_{0}}\right) q^{2(i+\alpha)+1}}\right)\right\} \\
& =-\frac{q}{\left(1-q^{2}\right)\left(t-\omega_{0}\right)}\left\{q^{\alpha}\left(t-\omega_{0}\right)^{\alpha} \prod_{i=0}^{\infty}\left(\frac{1-\left(\frac{\beta-\omega_{0}}{t-\omega_{0}}\right) q^{2 i}}{1-\left(\frac{\beta-\omega_{0}}{t-\omega_{0}}\right) q^{2(i+\alpha)}}\right)\right. \\
& \left.-\frac{\left(t-\omega_{0}\right)^{\alpha}}{q^{\alpha}} \prod_{i=0}^{\infty}\left(\frac{1-\left(\frac{\beta-\omega_{0}}{t-\omega_{0}}\right) q^{2(i+1)}}{1-\left(\frac{\beta-\omega_{0}}{t-\omega_{0}}\right) q^{2(i+\alpha+1)}}\right)\right\} \\
& =\frac{q^{1-\alpha}}{\left(1-q^{2}\right)}\left(t-\omega_{0}\right)^{\alpha-1}\left\{\frac{\prod_{i=0}^{\infty}\left(1-\left(\frac{\beta-\omega_{0}}{t-\omega_{0}}\right) q^{2(i+1)}\right)}{\prod_{i=0}^{\infty}\left(1-\left(\frac{\beta-\omega_{0}}{t-\omega_{0}}\right) q^{2(i+\alpha+1)}\right)}\right. \\
& \left.-q^{2 \alpha} \frac{\prod_{i=0}^{\infty}\left(1-\left(\frac{\beta-\omega_{0}}{t-\omega_{0}}\right) q^{2 i}\right)}{\prod_{i=0}^{\infty}\left(1-\left(\frac{\beta-\omega_{0}}{t-\omega_{0}}\right) q^{2(i+\alpha)}\right)}\right\} \\
& =\left(\frac{1-q^{2 \alpha}}{1-q^{2}}\right) q^{1-\alpha}\left(t-\omega_{0}\right)^{\alpha-1} \cdot \frac{1}{1-q^{2 \alpha}}\left\{\prod_{i=0}^{\infty}\left(\frac{1-\left(\frac{\beta-\omega_{0}}{t-\omega_{0}}\right) q^{2(i+1)}}{1-\left(\frac{\beta-\omega_{0}}{t-\omega_{0}}\right) q^{2(i+\alpha+1)}}\right)\right. \\
& \left.-q^{2 \alpha} \prod_{i=0}^{\infty}\left(\frac{1-\left(\frac{\beta-\omega_{0}}{t-\omega_{0}}\right) q^{2 i}}{1-\left(\frac{\beta-\omega_{0}}{t-\omega_{0}}\right) q^{2(i+\alpha)}}\right)\right\} \\
& =\widetilde{[\alpha]_{q}}\left(t-\omega_{0}\right)^{\alpha-1} q^{1-\alpha} \frac{\prod_{i=0}^{\infty}\left(1-\left(\frac{\beta-\omega_{0}}{t-\omega_{0}}\right) q^{2(i+1)}\right)}{\prod_{i=0}^{\infty}\left(1-\left(\frac{\beta-\omega_{0}}{t-\omega_{0}}\right) q^{2(i+\alpha)}\right)} \times \\
& \frac{\left(1-\left(\frac{\beta-\omega_{0}}{t-\omega_{0}}\right) q^{2 \alpha}\right)-q^{2 \alpha}\left(1-\left(\frac{\beta-\omega_{0}}{t-\omega_{0}}\right)\right)}{1-q^{2 \alpha}} \\
& =\widetilde{[\alpha]}]_{q}\left(t-\omega_{0}\right)^{\alpha-1} q^{1-\alpha} \prod_{i=0}^{\infty}\left(\frac{1-\left(\frac{\beta-\omega_{0}}{t-\omega_{0}}\right) q^{2(i+1)}}{1-\left(\frac{\beta-\omega_{0}}{t-\omega_{0}}\right) q^{2(i+\alpha)}}\right) \\
& =\widetilde{[\alpha]_{q}}\left(\rho_{q, \omega}(t)-\omega_{0}\right)^{\alpha-1} \prod_{i=0}^{\infty}\left(\frac{1-\left(\frac{\beta-\omega_{0}}{\rho_{q, \omega}(t)-\omega_{0}}\right) q^{2 i+1}}{1-\left(\frac{\beta-\omega_{0}}{\rho_{q, \omega}(t)-\omega_{0}}\right) q^{2(i+\alpha-1)+1}}\right) \\
& \left.=\widetilde{[\alpha]}_{q}\left(\rho_{q, \omega} \widetilde{(t)}-\beta\right)\right)_{q, \omega}^{\frac{\alpha-1}{}} .
\end{aligned}
$$


So, Lemma 4 (a) holds. Similarly to the above, we use Lemma 1 and Definition 1 to show that

$$
\tilde{D}_{q, \omega}\left(\beta-\widetilde{\rho_{q, \omega}}(t)\right)_{q, \omega}^{\underline{\alpha}}=-\widetilde{[\alpha]_{q}}(\widetilde{\beta-t})_{q, \omega}^{\frac{\alpha-1}{}}
$$

Then, Lemma 4 (b) holds.

Definition 2 ([20]). Let $I$ be any closed interval of $\mathbb{R}$ containing $a, b$ and $\omega_{0}$ and $f: I \rightarrow \mathbb{R}$ be $a$ given function. The symmetric Hahn integral of $f$ from $a$ to $b$ is defined by

$$
\int_{a}^{b} f(t) \tilde{d}_{q, \omega} t:=\int_{\omega_{0}}^{b} f(t) \tilde{d}_{q, \omega} t-\int_{\omega_{0}}^{a} f(t) \tilde{d}_{q, \omega} t
$$

where

$$
\tilde{\mathcal{I}}_{q, \omega} f(t)=\int_{\omega_{0}}^{x} f(t) \tilde{d}_{q, \omega} t:=\left(1-q^{2}\right)\left(x-\omega_{0}\right) \sum_{k=0}^{\infty} q^{2 k} f\left(\sigma_{q, \omega}^{2 k+1}(x)\right), \quad x \in I .
$$

Providing that the above series converges at $x=a$ and $x=b, f$ is called symmetric Hahn integrable on $[a, b]$. In addition, $f$ is symmetric Hahn integrable on I if it is symmetric Hahn integrable on $[a, b]$ for all $a, b \in I$.

For $N \in \mathbb{N}$, we define an operator $\tilde{\mathcal{I}}_{q, \omega}^{N}$ by

$$
[A] \quad \tilde{\mathcal{I}}_{q, \omega}^{0} f(x)=f(x) \text { and } \tilde{\mathcal{I}}_{q, \omega}^{N} f(x)=\tilde{\mathcal{I}}_{q, \omega} \tilde{\mathcal{I}}_{q, \omega}^{N-1} f(x), N \in \mathbb{N} .
$$

From the symmetric Hahn derivatives, we have

$$
[B] \quad \tilde{D}_{q, \omega} \tilde{\mathcal{I}}_{q, \omega} f(x)=f(x) \text { and } \tilde{\mathcal{I}}_{q, \omega} \tilde{D}_{q, \omega} f(x)=f(x)-f\left(\omega_{0}\right) .
$$

Lemma 5 ([20]). Properties of symmetric Hahn Integrals.

Let $0<q<1, \omega>0, a, b \in I_{q, \omega}^{T}$ and $f, g$ be symmetric Hahn integrable on $I_{q, \omega}^{T}$. Then,

(a) $\int_{a}^{a} f(t) \tilde{d}_{q, \omega} t=0$,

(b) $\quad \int_{a}^{b} f(t) \tilde{d}_{q, \omega} t=-\int_{b}^{a} f(t) \tilde{d}_{q, \omega} t$,

(c) $\quad \int_{a}^{b} f(t) \tilde{d}_{q, \omega} t=\int_{c}^{b} f(t) \tilde{d}_{q, \omega} t+\int_{a}^{c} f(t) \tilde{d}_{q, \omega} t, \quad c \in I_{q, \omega}^{T}, a<c<b$,

(d) $\quad \int_{a}^{b}[\alpha f(t)+\beta g(t)] \tilde{d}_{q, \omega} t=\alpha \int_{a}^{b} f(t) \tilde{d}_{q, \omega} t+\beta \int_{a}^{b} g(t) \tilde{d}_{q, \omega} t, \alpha, \beta \in \mathbb{R}$,

(e) $\quad \int_{a}^{b}\left[f\left(\rho_{q, \omega}(t)\right) \tilde{D}_{q, \omega} g(t)\right] \tilde{d}_{q, \omega} t=[f(t) g(t)]_{a}^{b}-\int_{a}^{b}\left[g\left(\sigma_{q, \omega}(t)\right) \tilde{D}_{q, \omega} f(t)\right] \tilde{d}_{q, \omega} t$.

We next introduce the fundamental theorem and Leibniz formula of symmetric Hahn calculus.

Lemma 6 ([20]). The fundamental theorem of symmetric Hahn calculus

Let $f: I \rightarrow \mathbb{R}$ be continuous at $\omega_{0}$. Then

$$
F(x):=\int_{\omega_{0}}^{x} f(t) \tilde{d}_{q, \omega} t, \quad x \in I
$$

is continuous at $\omega_{0}$ and $\tilde{D}_{q, \omega} F(x)$ exists for every $x \in \sigma_{q, \omega}(I):=\{q t+\omega: t \in I\}$ where

$$
\tilde{D}_{q, \omega} F(x)=f(x) \text {. }
$$

In addition,

$$
\int_{a}^{b} \tilde{D}_{q, \omega} f(t) \tilde{d}_{q, \omega} t=f(b)-f(a) \text { for all } a, b \in I
$$


Mathematics 2019, 7, 873

7 of 18

Lemma 7. The Leibniz formula of symmetric Hahn calculus

Let $f: I_{q, \omega}^{T} \times I_{q, \omega}^{T} \rightarrow \mathbb{R}$. Then,

$$
\tilde{D}_{q, \omega}\left[\int_{\omega_{0}}^{t} f(t, s) \tilde{d}_{q, \omega} s\right]=\int_{\omega_{0}}^{\rho_{q, \omega}(t)}{ }_{t} \tilde{D}_{q, \omega} f(t, s) \tilde{d}_{q, \omega} s+f\left(\sigma_{q, \omega}(t), t\right),
$$

where ${ }_{t} \tilde{D}_{q, \omega}$ is symmetric Hahn difference with respect to $t$.

Proof. For $t \in I_{q, \omega}^{T}$

$$
\begin{aligned}
& \tilde{D}_{q, \omega}\left[\int_{\omega_{0}}^{t} f(t, s) \tilde{d}_{q, \omega} s\right] \\
& =\frac{1}{\sigma_{q, \omega}(t)-\rho_{q, \omega}(t)}\left\{\int_{\omega_{0}}^{\sigma_{q, \omega}(t)} f\left(\sigma_{q, \omega}(t)\right) \tilde{d}_{q, \omega} s-\int_{\omega_{0}}^{\rho_{q, \omega}(t)} f\left(\rho_{q, \omega}(t), s\right) \tilde{d}_{q, \omega} s\right\} \\
& =\frac{1}{\sigma_{q, \omega}(t)-\rho_{q, \omega}(t)}\left\{\left[\int_{\omega_{0}}^{\sigma_{q, \omega}(t)} f\left(\sigma_{q, \omega}(t), s\right) \tilde{d}_{q, \omega} s-\int_{\omega_{0}}^{\rho_{q, \omega}(t)} f\left(\sigma_{q, \omega}(t), s\right) \tilde{d}_{q, \omega} s\right]\right. \\
& \left.+\left[\int_{\omega_{0}}^{\rho_{q, \omega}(t)} f\left(\sigma_{q, \omega}(t), s\right) \tilde{d}_{q, \omega} s-\int_{\omega_{0}}^{\rho_{q, \omega}(t)} f\left(\rho_{q, \omega}(t), s\right) \tilde{d}_{q, \omega} s\right]\right\} \\
& =\frac{1}{\sigma_{q, \omega}(t)-\rho_{q, \omega}(t)} \int_{\omega_{0}}^{\rho_{q, \omega}(t)}\left[f\left(\sigma_{q, \omega}(t), s\right)-f\left(\rho_{q, \omega}(t), s\right)\right] \tilde{d}_{q, \omega} s \\
& -\frac{q}{\left(1-q^{2}\right)\left(t-\omega_{0}\right)}\left\{\left(1-q^{2}\right)\left(\sigma_{q, \omega}(t)-\omega_{0}\right) \sum_{k=0}^{\infty} q^{2 k} f\left(\sigma_{q, \omega}(t), \sigma_{q, \omega}^{2 k+2}(t)\right)\right. \\
& \left.-\left(1-q^{2}\right)\left(\rho_{q, \omega}(t)-\omega_{0}\right) \sum_{k=0}^{\infty} q^{2 k} f\left(\sigma_{q, \omega}(t), \sigma_{q, \omega}^{2 k}(t)\right)\right\} \\
& =\int_{\omega_{0}}^{\rho_{q, \omega}(t)}{ }_{t} \tilde{D}_{q, \omega} f(t, s) \tilde{d}_{q, \omega} s \\
& -q\left\{\sum_{k=0}^{\infty} q^{2 k+1} f\left(\sigma_{q, \omega}(t), \sigma_{q, \omega}^{2 k+2}(t)\right)-\sum_{k=0}^{\infty} q^{2 k-1} f\left(\sigma_{q, \omega}(t), \sigma_{q, \omega}^{2 k}(t)\right)\right\} \\
& =\int_{\omega_{0}}^{\rho_{q, \omega}(t)}{ }_{t} \tilde{D}_{q, \omega} f(t, s) \tilde{d}_{q, \omega} s+f\left(\sigma_{q, \omega}(t), t\right) .
\end{aligned}
$$

The proof is complete.

Next, we give some auxiliary lemmas used for simplifying calculations.

Lemma 8. Let $0<q<1, \omega>0$ and $f: I \rightarrow \mathbb{R}$ be continuous at $\omega_{0}$. Then,

$$
\int_{\omega_{0}}^{t} \int_{\omega_{0}}^{r} f(s) \tilde{d}_{q, \omega} s \tilde{d}_{q, \omega} r=q \int_{\omega_{0}}^{t} \int_{q s+\omega}^{t} f(q s+\omega) \tilde{d}_{q, \omega} r \tilde{d}_{q, \omega} s .
$$


Mathematics 2019, 7, 873

8 of 18

Proof. From Definition 2, we find that

$$
\begin{aligned}
& \int_{\omega_{0}}^{t} \int_{\omega_{0}}^{r} f(s) \tilde{d}_{q, \omega} s \tilde{d}_{q, \omega} r \\
& =\int_{\omega_{0}}^{t}\left[\left(1-q^{2}\right)\left(r-\omega_{0}\right) \sum_{k=0}^{\infty} q^{2 k} f\left(\sigma_{q, \omega}^{2 k+1}(r)\right)\right] \tilde{d}_{q, \omega} r \\
& =\sum_{k=0}^{\infty} q^{2 k}\left(1-q^{2}\right)\left[\int_{\omega_{0}}^{t}\left(r-\omega_{0}\right) f\left(\sigma_{q, \omega}^{2 k+1}(r)\right) \tilde{d}_{q, \omega} r\right] \\
& =q\left(1-q^{2}\right)^{2}\left(t-\omega_{0}\right)^{2} \sum_{k=0}^{\infty} \sum_{m=0}^{\infty} q^{4 m+2 k} f\left(\sigma_{q, \omega}^{2 m+2 k+2}(t)\right) \\
& =q\left(1-q^{2}\right)^{2}\left(t-\omega_{0}\right)^{2} \sum_{k=0}^{\infty}\left[q^{4 m} f\left(\sigma_{q, \omega}^{2 m+2}(t)\right)+q^{4 m+2} f\left(\sigma_{q, \omega}^{2 m+4}(t)\right)+q^{4 m+4} f\left(\sigma_{q, \omega}^{2 m+6}(t)\right)+\ldots\right] \\
& =q\left(1-q^{2}\right)^{2}\left(t-\omega_{0}\right)^{2}\left\{\left[f\left(\sigma_{q, \omega}^{2}(t)\right)+q^{2} f\left(\sigma_{q, \omega}^{4}(t)\right)+q^{4} f\left(\sigma_{q, \omega}^{6}(t)\right)+\ldots\right]\right. \\
& +\left[q^{4} f\left(\sigma_{q, \omega}^{4}(t)\right)+q^{6} f\left(\sigma_{q, \omega}^{6}(t)\right)+q^{8} f\left(\sigma_{q, \omega}^{8}(t)\right)+\ldots\right] \\
& \left.+\left[q^{8} f\left(\sigma_{q, \omega}^{6}(t)\right)+q^{10} f\left(\sigma_{q, \omega}^{8}(t)\right)+q^{12} f\left(\sigma_{q, \omega}^{10}(t)\right)+\ldots\right]+\ldots\right\} \\
& =q\left(1-q^{2}\right)^{2}\left(t-\omega_{0}\right)^{2}\left\{f\left(\sigma_{q, \omega}^{2}(t)\right)+q^{2}\left(1+q^{2}\right) f\left(\sigma_{q, \omega}^{4}(t)\right)+q^{4}\left(1+q^{2}+q^{4}\right) f\left(\sigma_{q, \omega}^{6}(t)\right)+\ldots\right\} \\
& =q\left(1-q^{2}\right)^{2}\left(t-\omega_{0}\right)^{2} \sum_{k=0}^{\infty} q^{2 k}[\widetilde{k+1}]_{q} f\left(\sigma_{q, \omega}^{2 k+2}(t)\right) \\
& =q \int_{\omega_{0}}^{t}\left[t-\sigma_{q, \omega}(s)\right] f\left(\sigma_{q, \omega}(s)\right) \tilde{d}_{q, w} s \\
& =q \int_{\omega_{0}}^{t}\left[\int_{\omega_{0}}^{t} f\left(\sigma_{q, \omega}(s)\right) \tilde{d}_{q, w} r-\int_{\omega_{0}}^{\sigma_{q, \omega}(s)} f\left(\sigma_{q, \omega}(s)\right) \tilde{d}_{q, w} r\right] \tilde{d}_{q, w} s \\
& =q \int_{\omega_{0}}^{t} \int_{q s+\omega}^{t} f(q s+\omega) \tilde{d}_{q, \omega} r \tilde{d}_{q, \omega} s \text {. }
\end{aligned}
$$

In the next theorem we evaluate the multiple symmetric Hahn integrals as follows.

Theorem 1. For $f: I_{q, \omega}^{T} \rightarrow \mathbb{R}$, the multiple symmetric Hahn integral is given by

$$
\begin{aligned}
\tilde{\mathcal{I}}_{q, \omega}^{n} f(x) & :=\int_{\omega_{0}}^{x} \int_{\omega_{0}}^{\tau_{1}} \ldots \int_{\omega_{0}}^{\tau_{n-1}} f\left(\tau_{n}\right) \tilde{d}_{q, \omega} \tau_{n} \ldots \tilde{d}_{q, \omega} \tau_{2} \tilde{d}_{q, \omega} \tau_{1} \\
& =\frac{1}{\left[\widetilde{n-1]_{q} !}\right.} q^{\left(\begin{array}{l}
n \\
2
\end{array}\right)} \int_{\omega_{0}}^{t}(\widetilde{t-\tau})_{q, \omega}^{\frac{n-1}{q}} f\left(\sigma_{q, \omega}^{n-1}(\tau)\right) \tilde{d}_{q, \omega} \tau,
\end{aligned}
$$

where $n \in \mathbb{N}$ and $\left(\begin{array}{l}n \\ k\end{array}\right)=\frac{\Gamma(n+1)}{\Gamma(k+1) \Gamma(n-k+1)}$.

Proof. If $n=1, \tilde{\mathcal{I}}_{q, \omega} f(x)=\int_{\omega_{0}}^{x} f(\tau) \tilde{d}_{q, \omega} \tau$. 
Mathematics 2019, 7, 873

9 of 18

If $n=2$, by using Lemma 8 , we have

$$
\begin{aligned}
\tilde{\mathcal{I}}_{q, \omega}^{2} f(x) & =\int_{\omega_{0}}^{x} \int_{\omega_{0}}^{s} f(\tau) \tilde{d}_{q, \omega} \tau \tilde{d}_{q, \omega} s=q \int_{\omega_{0}}^{x} \int_{\sigma_{q, \omega}(\tau)}^{x} f\left(\sigma_{q, \omega}(\tau)\right) \tilde{d}_{q, \omega} s \tilde{d}_{q, \omega} \tau \\
& =q \int_{\omega_{0}}^{x}\left[x-\sigma_{q, \omega}(\tau)\right] f\left(\sigma_{q, \omega}(\tau)\right) \tilde{d}_{q, \omega} \tau \\
& \left.=q \int_{\omega_{0}}^{x}(\widetilde{x-\tau})_{q, \omega}^{\underline{1}} f(q \tau+\omega)\right) \tilde{d}_{q, \omega} \tau .
\end{aligned}
$$

We suppose that Theorem 1 holds for $n=k$ and then prove that it is true for $n=k+1$ as follows:

$$
\begin{aligned}
\tilde{\mathcal{I}}_{q, \omega}^{k+1} f(x)= & \left.\tilde{\mathcal{I}}_{q, \omega}\left[\frac{1}{[\widetilde{k-1}]_{q} !} q^{\left(\frac{k}{2}\right)} \int_{\omega_{0}}^{x} \widetilde{(x-\tau}\right)_{q, \omega}^{\frac{k-1}{2}} f\left(\sigma_{q, \omega}^{k-1}(\tau)\right) \tilde{d}_{q, \omega} \tau\right] \\
= & \frac{1}{[\widetilde{k-1}]_{q} !} q^{\left(\frac{k}{2}\right)} \int_{\omega_{0}}^{x} \int_{\omega_{0}}^{s}(\widetilde{s-\tau})_{q, \omega}^{\frac{k-1}{\omega}} f\left(\sigma_{q, \omega}^{k-1}(\tau)\right) \tilde{d}_{q, \omega} \tau \tilde{d}_{q, \omega} s \\
= & \frac{1}{[\widetilde{k-1}]_{q} !} q^{\left(\begin{array}{l}
k \\
2
\end{array}\right)}\left(1-q^{2}\right)\left(x-\omega_{0}\right) \sum_{m=0}^{\infty} q^{2 m}\left(1-q^{2}\right)\left[\sigma_{q, \omega}^{2 m+1}(x)-\omega_{0}\right] \times \\
& \sum_{l=0}^{\infty} q^{2 l}\left(\sigma_{q, \omega}^{2 m+1}(x) \widetilde{\sigma_{q, \omega}^{2 m+2 l+2}}(x)\right)_{q, \omega}^{\frac{k-1}{2 m}} f\left(\sigma_{q, \omega}^{k+2 m+2 l+1}(x)\right) .
\end{aligned}
$$

From (2), by using Lemma lb, we obtain

$$
\begin{aligned}
\tilde{\mathcal{I}}_{q, \omega}^{k+1} f(x)= & \frac{1}{[\widetilde{k-1}]_{q} !} q^{\left(\begin{array}{c}
k \\
2
\end{array}\right)}\left(1-q^{2}\right)^{2}\left(x-\omega_{0}\right) \sum_{m=0}^{\infty} \sum_{l=0}^{\infty} q^{4 m+2 l+1}\left(x-\omega_{0}\right) q^{(2 m+1)(k-1)} \times \\
& \left(x-\omega_{0}\right)^{k-1}\left(1-\widetilde{q^{2 l}}+1\right)_{q}^{\frac{k-1}{q}} f\left(\sigma_{q, \omega}^{k+2 m+2 l+1}(x)\right) \\
= & \frac{\widetilde{[k]}]_{q}}{\left.\widetilde{[k]_{q} !} q^{-k} q^{\left({ }^{k+1}\right.}\right)}\left(1-q^{2}\right)^{2}\left(x-\omega_{0}\right)^{k+1} \sum_{l=0}^{\infty} \sum_{m=0}^{l} q^{2 l} q^{(2 m+1) k} \times \\
& \left(1-\widetilde{q^{2 l-2 m+1}}\right)_{q}^{\frac{k-1}{q}} f\left(\sigma_{q, \omega}^{k+2 l+1}(x)\right) .
\end{aligned}
$$

From (2), by using Lemma la, we obtain

$$
\begin{aligned}
& \tilde{\mathcal{I}}_{q, \omega}^{k+1} f(x)=\frac{1}{\left.\widetilde{[k]}]_{q} q^{\left({ }^{k+1}\right.}\right)} \int_{\omega_{0}}^{x}(\widetilde{x-\tau})_{q, \omega}^{\underline{k}} f\left(\sigma_{q, \omega}^{k}(\tau)\right) \tilde{d}_{q, \omega} \tau \\
& =\frac{1}{\widetilde{[k]_{q}} !} q^{\left(\frac{k+1}{2}\right)}\left(1-q^{2}\right)\left(x-\omega_{0}\right) \sum_{l=0}^{\infty} q^{2 l}\left(x-\widetilde{\sigma_{q, \omega}^{2 l+1}}(x)\right)_{q, \omega}^{\underline{k}} f\left(\sigma_{q, \omega}^{k+2 l+1}(x)\right)
\end{aligned}
$$

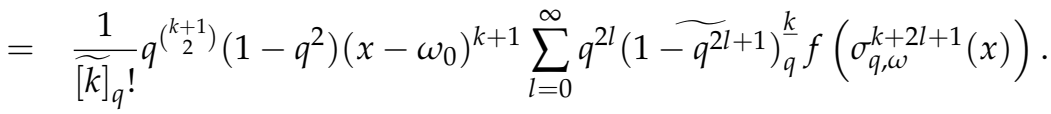

Since 


$$
\begin{aligned}
& \widetilde{[k]} q^{-k}\left(1-q^{2}\right) \sum_{m=0}^{l} q^{(2 m+1) k}\left(1-\widetilde{q^{2 l-2 m+1}}\right)_{q}^{\frac{k-1}{q}} \\
& =\left(1-q^{2 k}\right) \sum_{m=0}^{l} q^{2 m k}\left(1-\widetilde{q^{2 l-2 m+1}}\right)_{q}^{\frac{k-1}{q}} \\
& =\left(1-q^{2 k}\right)\left\{\prod_{i=0}^{k-2}\left[1-q^{2(l+i-1)}\right]+\ldots+q^{2(l-2) k} \prod_{i=0}^{k-2}\left[1-q^{2 i+6}\right]+q^{2(l-1) k} \prod_{i=0}^{k-2}\left[1-q^{2 i+4}\right]\right. \\
& \left.+q^{2 l k} \prod_{i=0}^{k-2}\left[1-q^{2 i+2}\right]\right\} \\
& =\left(1-q^{2 k}\right)\left\{\prod_{i=0}^{k-2}\left[1-q^{2(l+i-1)}\right]+\ldots+q^{2(l-2) k} \prod_{i=0}^{k-2}\left[1-q^{2 i+6}\right]\right. \\
& \left.+q^{2(l-1) k}\left(1-q^{2 k+2}\right) \prod_{i=0}^{k-3}\left[1-q^{2 i+4}\right]\right\} \\
& =\left(1-q^{2 k}\right)\left\{\prod_{i=0}^{k-2}\left[1-q^{2(l+i-1)}\right]+\ldots+q^{2(l-3) k} \prod_{i=0}^{k-2}\left[1-q^{2 i+8}\right]\right. \\
& \left.+q^{2(l-2) k}\left[\prod_{i=0}^{k-2}\left[1-q^{2 i+6}\right]+q^{2 k}\left(1-q^{2 k+2}\right) \prod_{i=0}^{k-3}\left[1-q^{2 i+4}\right]\right]\right\} \\
& =\left(1-q^{2 k}\right)\left\{\prod_{i=0}^{k-2}\left[1-q^{2(l+i-1)}\right]+\ldots+q^{2(l-3) k} \prod_{i=0}^{k-2}\left[1-q^{2 i+8}\right]\right. \\
& \left.+q^{2(l-2) k}\left(1-q^{2 k+4}\right)\left(1-q^{2 k+2}\right) \prod_{i=0}^{k-4}\left[1-q^{2 i+6}\right]\right\} \\
& =\left(1-q^{2 k}\right)\left\{\prod_{i=0}^{k-2}\left[1-q^{2(l+i-1)}\right]+\ldots+q^{2 k}\left(1-q^{2(l+k-1)}\right)\left(1-q^{2(l+k-2)}\right) \ldots \times\right. \\
& \left.\left(1-q^{2 k+2}\right) \prod_{i=0}^{k-(l+1)}\left[1-q^{2(l+i)}\right]\right\} \\
& =\left(1-q^{2 k+2 l)}\right)\left(1-q^{2 k+2 l-2)}\right) \ldots\left(1-q^{2 k+2}\right)\left(1-q^{2 k}\right)\left(1-q^{2 k-2}\right) \ldots\left(1-q^{2 l+4}\right)\left(1-q^{2 l+2}\right) \\
& =\prod_{i=0}^{k-1}\left(1-q^{2(l+i+1)}\right) \\
& =\left(1-\widetilde{q^{2 l+1}}(x)\right)_{q}^{\underline{k}}
\end{aligned}
$$

We find that (1) holds when $n=k+1$.

Our proof is done using mathematical induction.

\section{Fractional Symmetric Hahn Integral}

In Section 2, we have presented the multiple symmetric Hahn integral for integer order in the form (1). We next apply this result for fractional orders that can be used to further define fractional symmetric Hahn difference operators of Riemann-Liouville and Caputo types. We first introduce the fractional symmetric Hahn integral as follows. 
Definition 3. Let $\alpha, \omega>0,0<q<1$, and $f$ be a function defined on $I_{q, \omega}^{T}$. The fractional symmetric Hahn integral is defined by

$$
\begin{aligned}
\widetilde{\mathcal{I}}_{q, \omega}^{\alpha} f(t) & :=\frac{q^{\left(\begin{array}{c}
\alpha \\
2
\end{array}\right)}}{\widetilde{\Gamma}_{q}(\alpha)} \int_{\omega_{0}}^{t}(\widetilde{t-s})_{q, \omega}^{\frac{\alpha-1}{\omega}} f\left(\sigma_{q, \omega}^{\alpha-1}(s)\right) \tilde{d}_{q, \omega} s \\
& =\frac{\left(1-q^{2}\right) q^{\left(\begin{array}{c}
\alpha \\
2
\end{array}\right)}\left(t-\omega_{0}\right)}{\widetilde{\Gamma}_{q}(\alpha)} \sum_{k=0}^{\infty} q^{2 k}\left(t-\sigma_{q, \omega}^{2 k+1}(t)\right)_{q, \omega}^{\frac{\alpha-1}{}} f\left(\sigma_{q, \omega}^{2 k+\alpha}(t)\right),
\end{aligned}
$$

and $\left.\widetilde{\mathcal{I}}_{q, \omega}^{0} f\right)(t)=f(t)$.

By Lemma 1a, $\left(t-\widetilde{\sigma^{2} k+1}{ }_{q, \omega}(t)\right) \frac{\alpha-1}{q, \omega}=\left(t-\omega_{0}\right)^{\alpha-1}\left(1-\widetilde{q^{2 k}+1}\right)_{q}^{\frac{\alpha-1}{}}$. It implies that

$$
\widetilde{\mathcal{I}}_{q, \omega}^{\alpha} f(t)=\frac{\left(1-q^{2}\right) q^{\left(\begin{array}{c}
\alpha \\
2
\end{array}\right)}\left(t-\omega_{0}\right)^{\alpha}}{\tilde{\Gamma}_{q}(\alpha)} \sum_{k=0}^{\infty} q^{2 k}\left(1-\widetilde{q^{2 k+1}}\right)_{q}^{\underline{\alpha-1}} f\left(\sigma_{q, \omega}^{2 k+\alpha}(t)\right) .
$$

Some properties of the fractional symmetric Hahn integral are given below.

Theorem 2. For $\alpha, \omega>0,0<q<1$, and $f: I_{q, \omega}^{T} \rightarrow \mathbb{R}$,

$$
\widetilde{\mathcal{I}}_{q, \omega}^{\alpha} f(t)=\widetilde{\mathcal{I}}_{q, \omega}^{\alpha+1}\left[\widetilde{D}_{q, \omega} f(t)\right]+\frac{f\left(\omega_{0}\right)}{\widetilde{\Gamma}_{q}(\alpha+1)} q^{\left(\begin{array}{c}
\alpha \\
2
\end{array}\right)}\left(t-\omega_{0}\right)^{\alpha} .
$$

Proof. We apply Lemma 4 b and Lemma 5e to (5). Then, we get

$$
\begin{aligned}
\widetilde{\mathcal{I}}_{q, \omega}^{\alpha} f(t) & :=\frac{q^{\left(\begin{array}{c}
\alpha \\
2
\end{array}\right)}}{\widetilde{\Gamma}_{q}(\alpha)} \int_{\omega_{0}}^{t}(\widetilde{t-s})_{q, \omega}^{\frac{\alpha-1}{q}} f\left(\sigma_{q, \omega}^{\alpha-1}(s)\right) \tilde{d}_{q, \omega} s \\
= & -\frac{q^{\left(\begin{array}{c}
\alpha \\
2
\end{array}\right)}}{\widetilde{\Gamma}_{q}(\alpha) \widetilde{[\alpha]_{q}}} \int_{\omega_{0}}^{t} f\left(\rho_{q, \omega}\left(\sigma_{q, \omega}^{\alpha}(s)\right)\right) \tilde{D}_{q, \omega}\left(t-\widetilde{\rho_{q, \omega}}(s)\right)_{q, \omega}^{\underline{\alpha}} \tilde{d}_{q, \omega} s \\
= & \frac{q^{\left(\begin{array}{c}
\alpha \\
2
\end{array}\right)}}{\widetilde{\Gamma}_{q}(\alpha+1)}\left\{-\left[\left(t-\widetilde{\rho_{q, \omega}}(s)\right)_{q, \omega}^{\underline{\alpha}} f\left(\sigma_{q, \omega}^{\alpha}(s)\right)\right]_{\omega_{0}}^{t}+\right. \\
& \left.q^{\alpha} \int_{\omega_{0}}^{t} \tilde{D}_{q, \omega} f\left(\sigma_{q, \omega}^{\alpha}(s)\right)(\widetilde{t-s})_{q, \omega}^{\underline{\alpha}} \tilde{d}_{q, \omega} s\right\} \\
= & \widetilde{\mathcal{I}}_{q, \omega}^{\alpha+1}\left[\widetilde{D}_{q, \omega} f(t)\right]+\frac{f\left(\omega_{0}\right)}{\widetilde{\Gamma}_{q}(\alpha+1)} q^{\left(\begin{array}{c}
\alpha \\
2
\end{array}\right)}\left(t-\omega_{0}\right)^{\alpha} .
\end{aligned}
$$

Theorem 3. For $\alpha, \beta, \omega>0,0<q<1, f: I_{q, \omega}^{T} \rightarrow \mathbb{R}$, and $a \in I_{q, \omega}^{T}$,

$$
\int_{\omega_{0}}^{a}(\widetilde{t-s}) \frac{\beta-1}{q, \omega} \widetilde{\mathcal{I}}_{q, \omega}^{\alpha} f(s) \tilde{d}_{q, \omega} s=0
$$


Proof. From Definition 3, for $n \in \mathbb{N}_{0}$, we have

$$
\begin{aligned}
\widetilde{\mathcal{I}}_{q, \omega}^{\alpha} f\left(\sigma_{q, \omega}^{2 n+1}(a)\right)= & \frac{q^{\left(\frac{\alpha}{2}\right)}}{\widetilde{\Gamma}_{q}(\alpha)} \int_{\omega_{0}}^{\sigma_{q, \omega}^{2 n+1}(a)}\left(\sigma_{q, \omega}^{2 n+\widetilde{1}(a)}-s\right)_{q, \omega}^{\frac{\alpha-1}{\alpha}} f\left(\sigma_{q, \omega}^{\alpha-1}(s)\right) \tilde{d}_{q, \omega} s \\
= & \frac{\left(1-q^{2}\right) q^{\left(\frac{\alpha}{2}\right)}\left[\sigma_{q, \omega}^{2 n+1}(a)-\omega_{0}\right]}{\widetilde{\Gamma}_{q}(\alpha)} \sum_{k=0}^{\infty} q^{2 k}\left(\sigma_{q, \omega}^{2 n+1}(a) \widetilde{\sigma_{q, \omega}^{2 k+2 n+2}}(a)\right)_{q, \omega}^{\frac{\alpha-1}{\omega}} \times \\
& f\left(\sigma_{q, \omega}^{2 k+\alpha}(a)\right) .
\end{aligned}
$$

By using Lemma 2, we find that $\left(\sigma_{q, \omega}^{2 n+1}(a)-\widetilde{\sigma} q, \omega^{2 k+2 n+2}(a)\right)_{q, \omega}^{\frac{\alpha-1}{}}=0$. Therefore,

$$
\widetilde{\mathcal{I}}_{q, \omega}^{\alpha} f\left(\sigma_{q, \omega}^{2 n+1}(a)\right)=0 .
$$

From Definition 2 and (7), we have

$$
\begin{aligned}
& \int_{\omega_{0}}^{a}(\widetilde{t-s}) \frac{\beta-1}{q, \omega} \widetilde{\mathcal{I}}_{q, \omega}^{\alpha} f(s) \tilde{d}_{q, \omega} s \\
= & \left(1-q^{2}\right)\left(a-\omega_{0}\right) \sum_{k=0}^{\infty} q^{2 k}\left(t-\widetilde{\sigma_{q, \omega}^{2 k+1}}(a)\right)_{q, \omega}^{\frac{\beta-1}{w}}\left[\widetilde{\mathcal{I}}_{q, \omega}^{\alpha} f\left(\sigma_{q, \omega}^{2 k+1}(a)\right)\right]=0 .
\end{aligned}
$$

Lemma 9 ([22]). For $\mu, \alpha, \beta>\in \mathbb{R}^{+}$, the following identity is valid:

$$
\sum_{k=0}^{\infty} q^{\alpha k} \frac{\left(1-\mu q^{1-k}\right) \frac{\alpha-1}{q}\left(1-\mu q^{1+k}\right) \frac{\beta-1}{q}}{(1-q) \frac{\alpha-1}{q}(1-q) \frac{\beta-1}{q}}=\frac{(1-\mu q) \frac{\alpha+\beta-1}{q}}{(1-q) \frac{\alpha+\beta-1}{q}} .
$$

Theorem 4. For $\alpha, \beta, \omega>0,0<q<1$, and $f: I_{q, \omega}^{T} \rightarrow \mathbb{R}$,

$$
\widetilde{\mathcal{I}}_{q, \omega}^{\alpha}\left[\widetilde{\mathcal{I}}_{q, \omega}^{\beta} f(t)\right]=\widetilde{\mathcal{I}}_{q, \omega}^{\beta}\left[\widetilde{\mathcal{I}}_{q, \omega}^{\alpha} f(t)\right]=\widetilde{\mathcal{I}}_{q, \omega}^{\alpha+\beta} f(t) .
$$


Proof. By Definition 3, for $t \in I_{q, \omega}^{T}$, we have

$$
\begin{aligned}
& \widetilde{\mathcal{I}}_{q, \omega}^{\alpha} \widetilde{\mathcal{I}}_{q, \omega}^{\beta} f(t)=\widetilde{\mathcal{I}}_{q, \omega}^{\alpha}\left[\frac{q^{\left(\frac{\beta}{2}\right)}}{\widetilde{\Gamma}_{q}(\beta)} \int_{\omega_{0}}^{t}(\widetilde{t-s})_{q, \omega}^{\frac{\beta-1}{q}} f\left(\sigma_{q, \omega}^{\beta-1}(s)\right) \tilde{d}_{q, \omega} s\right] \\
& =\frac{q^{\left(\begin{array}{c}
\alpha \\
2
\end{array}\right)+\left(\begin{array}{c}
\beta \\
2
\end{array}\right)}}{\widetilde{\Gamma}_{q}(\alpha) \widetilde{\Gamma}_{q}(\beta)} \int_{\omega_{0}}^{t}(\widetilde{t-x})_{q, \omega}^{\frac{\alpha-1}{\sigma^{2}}} \int_{\omega_{0}}^{\sigma_{q, \omega}^{\alpha-1}(x)}\left(\sigma_{q, \omega}^{\alpha-1} \widetilde{(x)}-s\right)_{q, \omega}^{\frac{\beta-1}{q}} f\left(\sigma_{q, \omega}^{\beta-1}(s)\right) \tilde{d}_{q, \omega} s \tilde{d}_{q, \omega} x \\
& =\frac{q^{\left(\begin{array}{c}
\alpha \\
2
\end{array}\right)+\left(\begin{array}{c}
\beta \\
2
\end{array}\right)+\alpha \beta}}{\widetilde{\Gamma}_{q}(\alpha) \widetilde{\Gamma}_{q}(\beta)}\left(1-q^{2}\right)^{2}\left(t-\omega_{0}\right)^{\alpha+\beta} \times \\
& \sum_{k=0}^{\infty} \sum_{h=0}^{\infty} q^{2 k+2 h+2 k \beta}\left(1-\widetilde{q^{2 k}+1}\right)^{\frac{\alpha-1}{q}}\left(1-\widetilde{q^{2 h}+1}\right) \frac{\beta-1}{q} f\left(\sigma_{q, \omega}^{2 h+2 k+\alpha+\beta}(t)\right) \\
& =\frac{\left.q^{(\alpha+\beta}\right)}{\widetilde{\Gamma}_{q}(\alpha) \widetilde{\Gamma}_{q}(\beta)}\left(1-q^{2}\right)^{2}\left(t-\omega_{0}\right)^{\alpha+\beta} \times \\
& \sum_{k=0}^{\infty} \sum_{h=k}^{\infty} q^{2 h+2 k \beta}\left(1-\widetilde{q^{2 k}+1}\right)_{q}^{\frac{\alpha-1}{q}}\left(1-\widetilde{q^{2 h-}-2 k+1}\right) \frac{\beta-1}{q} f\left(\sigma_{q, \omega}^{2 h+\alpha+\beta}(t)\right) \\
& =\frac{q^{\left(\begin{array}{c}
\alpha+\beta \\
2
\end{array}\right)}}{\widetilde{\Gamma}_{q}(\alpha) \widetilde{\Gamma}_{q}(\beta)}\left(1-q^{2}\right)^{2}\left(t-\omega_{0}\right)^{\alpha+\beta} \times \\
& \sum_{h=0}^{\infty} q^{2 h}\left[\sum_{k=0}^{h} q^{2 k \beta}\left(1-\widetilde{q^{2 k}+1}\right)_{q}^{\frac{\alpha-1}{q}}\left(1-\widetilde{q^{2 h-2 k+1}}\right)_{q}^{\frac{\beta-1}{q}}\right] f\left(\sigma_{q, \omega}^{2 h+\alpha+\beta}(t)\right) .
\end{aligned}
$$

Using [21] (Theorem 2), Lemma 9, and $\widetilde{\Gamma}_{q}(\alpha+\beta)=\frac{(\widetilde{1-q}) \frac{\alpha+\beta-1}{q}}{\left(1-q^{2}\right)^{\alpha+\beta-1}}$, we obtain

$$
\begin{aligned}
\sum_{k=0}^{h} q^{2 k \beta}\left(1-\widetilde{q^{2 k}+1}\right)_{q}^{\frac{\alpha-1}{q}}\left(1-\widetilde{q^{2 h}-2 k+1}\right)^{\frac{\beta-1}{q}} & \left.=\widetilde{\left(1-q^{2}\right.}\right)_{q}^{\frac{\alpha-1}{q}}\left(\widetilde{1-q^{2}}\right)^{\frac{\beta-1}{q}} \frac{\left(1-\widetilde{q^{2 h}+1}\right) \frac{\alpha+\beta-1}{q}}{\left(1-q^{2}\right) \frac{\alpha+\beta-1}{q}} \\
& =\frac{\widetilde{\Gamma}_{q}(\alpha) \widetilde{\Gamma}_{q}(\beta)}{\left(1-q^{2}\right) \widetilde{\Gamma}_{q}(\alpha+\beta)}\left(1-\widetilde{q^{2 h}+1}\right) \frac{\alpha+\beta-1}{q} .
\end{aligned}
$$

Therefore,

$$
\begin{aligned}
\widetilde{\mathcal{I}}_{q, \omega}^{\alpha} \widetilde{\mathcal{I}}_{q, \omega}^{\beta} f(t) & =\frac{q^{\left(\begin{array}{c}
\alpha+\beta \\
2
\end{array}\right)}}{\widetilde{\Gamma}_{q}(\alpha+\beta)}\left(1-q^{2}\right)\left(t-\omega_{0}\right)^{\alpha+\beta} \sum_{h=0}^{\infty} q^{2 h}\left(1-\widetilde{q^{2 h}+1}\right) \frac{\alpha+\beta-1}{q} f\left(\sigma_{q, \omega}^{2 h+\alpha+\beta}(t)\right) \\
& =\frac{q^{\left(\begin{array}{c}
\alpha+\beta \\
2
\end{array}\right)}}{\widetilde{\Gamma}_{q}(\alpha+\beta)}\left(1-q^{2}\right)\left(t-\omega_{0}\right) \sum_{h=0}^{\infty} q^{2 h}\left(t-\widetilde{\sigma_{q, \omega}^{2 h+1}}(t)\right)_{q, \omega}^{\frac{\alpha+\beta-1}{q}} f\left(\sigma_{q, \omega}^{2 h+\alpha+\beta}(t)\right) \\
& =\frac{q^{\left(\begin{array}{c}
\alpha+\beta \\
2
\end{array}\right)}}{\widetilde{\Gamma}_{q}(\alpha+\beta)} \int_{0}^{t} \widetilde{(t-s)} \frac{\alpha+\beta-1}{q, \omega} f\left(\sigma_{q, \omega}^{\alpha+\beta-1}(s)\right) \tilde{d}_{q, \omega} s=\widetilde{\mathcal{I}}_{q, \omega}^{\alpha+\beta} f(t) .
\end{aligned}
$$

Similarly to the above, by commuting the order of integrals, we have

$$
\widetilde{\mathcal{I}}_{q, \omega}^{\beta} \widetilde{\mathcal{I}}_{q, \omega}^{\alpha} f(t)=\widetilde{\mathcal{I}}_{q, \omega}^{\alpha+\beta} f(t) .
$$

\section{The Fractional Symmetric Hahn Difference Operator of the Riemann-Liouville Type}

In this section, we introduce the fractional symmetric Hahn difference operator of Riemann-Liouville as given in the following definition. 
Definition 4. For $\alpha, \omega>0,0<q<1$ and $f$ defined on $I_{q, \omega}^{T}$, the fractional symmetric Hahn difference operator of Riemann-Liouville type of order $\alpha$ is defined by

$$
\begin{aligned}
& \widetilde{D}_{q, \omega}^{\alpha} f(t):=\widetilde{D}_{q, \omega}^{N} \widetilde{\mathcal{I}}_{q, \omega}^{N-\alpha} f(t), \\
& \widetilde{D}_{q, \omega}^{0} f(t)=f(t)
\end{aligned}
$$

where $N-1<\alpha<N, N \in \mathbb{N}$.

Next, we will establish some properties of fractional symmetric Hahn difference operators of the Riemann-Liouville type as follows.

Theorem 5. For $\alpha, \omega>0,0<q<1$ and $f: I_{q, \omega}^{T} \rightarrow \mathbb{R}$,

$$
\tilde{D}_{q, \omega}^{\alpha} \widetilde{\mathcal{I}}_{q, \omega}^{\alpha} f(t)=f(t)
$$

Proof. For some $N-1<\alpha<N, N \in \mathbb{N}$, we find that

$$
\tilde{D}_{q, \omega}^{\alpha} \mathcal{I}_{q, \omega}^{\alpha} f(t)=\tilde{D}_{q, \omega}^{N} \tilde{\mathcal{I}}_{q, \omega}^{N-\alpha} \tilde{\mathcal{I}}_{q, \omega}^{\alpha} f(t)=\tilde{D}_{q, \omega}^{N} \tilde{\mathcal{I}}_{q, \omega}^{N} f(t)=f(t) .
$$

The proof is complete.

Theorem 6. For $\alpha \in(0,1), \omega>0,0<q<1$ and $f: I_{q, \omega}^{T} \rightarrow \mathbb{R}$,

$$
\widetilde{\mathcal{I}}_{q, \omega}^{\alpha} \tilde{D}_{q, \omega}^{\alpha} f(t)=f(t)+C\left(t-\omega_{0}\right)^{\alpha-1}, C \in \mathbb{R} .
$$

Proof. Let $C(t)=\widetilde{\mathcal{I}}_{q, \omega}^{\alpha} \tilde{D}_{q, \omega}^{\alpha} f(t)-f(t)$. Taking $\tilde{D}_{q, \omega}^{\alpha}$ to both sides and using Theorem 5 , we have

$$
\tilde{D}_{q, \omega}^{\alpha} C(t)=\tilde{D}_{q, \omega}^{\alpha} \widetilde{\mathcal{I}}_{q, \omega}^{\alpha} \tilde{D}_{q, \omega}^{\alpha} f(t)-\tilde{D}_{q, \omega}^{\alpha} f(t)=\tilde{D}_{q, \omega}^{\alpha} f(t)-\tilde{D}_{q, \omega}^{\alpha} f(t)=0 .
$$

From

$$
\begin{aligned}
& \int_{\omega_{0}}^{t}(\widetilde{t-s})_{q, \omega}^{\frac{-\alpha}{\alpha}}\left(s-\omega_{0}\right)^{\alpha-1} \tilde{d}_{q, \omega} s \\
= & \left(1-q^{2}\right)\left(t-\omega_{0}\right) \sum_{k=0}^{\infty} q^{2 k}\left(t-\widetilde{\sigma_{q, \omega}^{2 k+1}}(t)\right)_{q, \omega}^{\frac{-\alpha}{\alpha}}\left(\sigma_{q, \omega}^{2 k+1}(t)-\omega_{0}\right)^{\alpha-1} \\
= & q^{\alpha-1}\left(1-q^{2}\right) \sum_{k=0}^{\infty} q^{2 \alpha k}\left(1-\widetilde{q^{2 k}+1}\right)_{q}^{-\alpha}
\end{aligned}
$$

and according to Definitions 3 and 4, we have

$$
\begin{aligned}
& \tilde{D}_{q, \omega}^{\alpha}\left(t-\omega_{0}\right)^{\alpha-1} \\
= & \tilde{D}_{q, \omega} \tilde{I}_{q, \omega}^{1-\alpha}\left(t-\omega_{0}\right)^{\alpha-1} \\
= & \tilde{D}_{q, \omega}\left[\frac{q^{\left(\frac{1-\alpha}{2}\right)}}{\widetilde{\Gamma}_{q}(1-\alpha)} \int_{\omega_{0}}^{t}(\widetilde{t-s})_{q, \omega}^{\frac{-\alpha}{\omega}}\left(\sigma_{q, \omega}^{-\alpha}(s)-\omega_{0}\right)^{\alpha-1} \tilde{d}_{q, \omega} s\right] \\
= & \tilde{D}_{q, \omega}\left[\frac{q^{\left(\frac{1-\alpha}{2}\right)}\left(1-q^{2}\right)\left(t-\omega_{0}\right)}{\widetilde{\Gamma}_{q}(1-\alpha)} \sum_{k=0}^{\infty} q^{2 k}\left(t-\widetilde{\sigma_{q, \omega}^{2 k+1}}(t)\right)_{q, \omega}^{\frac{-\alpha}{(1-\alpha}}\left(\sigma_{q, \omega}^{2 k-\alpha+1}(t)-\omega_{0}\right)^{\alpha-1}\right] \\
= & \tilde{D}_{q, \omega}\left[\frac{q^{\left(\frac{1-\alpha}{2}\right)-(\alpha-1)^{2}}\left(1-q^{2}\right)}{\widetilde{\Gamma}_{q}(1-\alpha)} \sum_{k=0}^{\infty} q^{2 k \alpha}\left(1-\widetilde{\sigma_{q, \omega}^{2 k+1}}\right)_{q, \omega}^{\frac{-\alpha}{2}}\right] \\
= & 0 .
\end{aligned}
$$


Hence, $C(t)=C\left(t-\omega_{0}\right)^{\alpha-1}$.

Theorem 7. Let $\alpha, \omega>0,0<q<1$ and $f: I_{q, \omega}^{T} \rightarrow \mathbb{R}$. Then,

$$
\tilde{\mathcal{I}}_{q, \omega}^{\alpha} \tilde{D}_{q, \omega}^{\alpha} f(t)=f(t)+C_{1}\left(t-\omega_{0}\right)^{\alpha-1}+C_{2}\left(t-\omega_{0}\right)^{\alpha-2}+\ldots+C_{N}\left(t-\omega_{0}\right)^{\alpha-N}
$$

for some $C_{i} \in \mathbb{R}, i=1,2, \ldots, N$ and $N-1<\alpha<N$ for $N \in \mathbb{N}$.

Proof. By Theorem 2, we have

$$
\begin{aligned}
& \tilde{\mathcal{I}}_{q, \omega}^{\alpha} \tilde{D}_{q, \omega}^{\alpha} f(t)=\tilde{\mathcal{I}}_{q, \omega}^{\alpha} \tilde{D}_{q, \omega}^{N} \tilde{\mathcal{I}}_{q, \omega}^{N-\alpha} f(t) \\
& =\quad \tilde{\mathcal{I}}_{q, \omega}^{\alpha-1} \tilde{D}_{q, \omega}^{N-1} \tilde{\mathcal{I}}_{q, \omega}^{N-\alpha} f(t)-\frac{\left.q^{(\alpha-1}\right)}{\widetilde{\Gamma}_{q}(\alpha)}\left(t-\omega_{0}\right)^{\alpha-1} \tilde{D}_{q, \omega}^{N-1} \tilde{\mathcal{I}}_{q, \omega}^{N-\alpha} f\left(\omega_{0}\right) \\
& =\quad \tilde{\mathcal{I}}_{q, \omega}^{\alpha-2} \tilde{D}_{q, \omega}^{N-2} \tilde{\mathcal{I}}_{q, \omega}^{N-\alpha} f(t)-\frac{q^{(\alpha-2)}}{\widetilde{\Gamma}_{q}(\alpha-1)}\left(t-\omega_{0}\right)^{\alpha-2} \tilde{D}_{q, \omega}^{N-2} \tilde{\mathcal{I}}_{q, \omega}^{N-\alpha} f\left(\omega_{0}\right) \\
& -\frac{q^{\left(\begin{array}{c}
\alpha-1 \\
2
\end{array}\right)}}{\widetilde{\Gamma}_{q}(\alpha)}\left(t-\omega_{0}\right)^{\alpha-1} \tilde{D}_{q, \omega}^{N-1} \tilde{\mathcal{I}}_{q, \omega}^{N-\alpha} f\left(\omega_{0}\right) \\
& \text { - } \\
& =\tilde{\mathcal{I}}_{q, \omega}^{\alpha-N+1} \tilde{D}_{q, \omega}^{\alpha-N+1} f(t)-\frac{\left.q^{(\alpha-N+1}\right)}{\widetilde{\Gamma}_{q}(\alpha-N+2)}\left(t-\omega_{0}\right)^{\alpha-N+1} \tilde{D}_{q, \omega} \tilde{\mathcal{I}}_{q, \omega}^{N-\alpha} f\left(\omega_{0}\right) \\
& -\ldots-\frac{q^{\left(\frac{\alpha-2}{2}\right)}}{\widetilde{\Gamma}_{q}(\alpha-1)}\left(t-\omega_{0}\right)^{\alpha-2} \tilde{D}_{q, \omega}^{N-2} \tilde{\mathcal{I}}_{q, \omega}^{N-\alpha} f\left(\omega_{0}\right) \\
& -\frac{q^{\left(\begin{array}{c}
\alpha-1 \\
2
\end{array}\right)}}{\widetilde{\Gamma}_{q}(\alpha)}\left(t-\omega_{0}\right)^{\alpha-1} \tilde{D}_{q, \omega}^{N-1} \tilde{\mathcal{I}}_{q, \omega}^{N-\alpha} f\left(\omega_{0}\right) .
\end{aligned}
$$

Using Theorem 6, we obtain

$$
\tilde{\mathcal{I}}_{q, \omega}^{\alpha} \tilde{D}_{q, \omega}^{\alpha} f(t)=f(t)+C_{1}\left(t-\omega_{0}\right)^{\alpha-1}+C_{2}\left(t-\omega_{0}\right)^{\alpha-2}+\ldots+C_{N}\left(t-\omega_{0}\right)^{\alpha-N} .
$$

The proof is complete.

Corollary 1. Let $\alpha, \omega>0,0<q<1$ and $f: I_{q, \omega}^{T} \rightarrow \mathbb{R}$. Then,

$$
\tilde{\mathcal{I}}_{q, \omega}^{\alpha} \tilde{D}_{q, \omega}^{\alpha} f(t)=f(t)-\sum_{k=0}^{N-1} \frac{\left.\left(t-\omega_{0}\right)^{\alpha-N+k} q^{(\alpha-N+k}\right)}{\tilde{\Gamma}_{q}(\alpha-N+k+1)}\left[\tilde{D}_{q, \omega}^{\alpha-N+k} f\left(\omega_{0}\right)\right]
$$

where $N-1<\alpha<N$ for $N \in \mathbb{N}$.

\section{The Fractional Symmetric Hahn Difference Operator of the Caputo type}

Finally, we introduce the fractional symmetric Hahn difference operator of Caputo types as follows. 
Definition 5. For $\alpha, \omega>0,0<q<1$ and $f: I_{q, \omega}^{T} \rightarrow \mathbb{R}$, the fractional symmetric Hahn difference operator of Caputo type of order $\alpha$ is defined by

$$
\begin{aligned}
{ }^{C} \tilde{D}_{q, \omega}^{\alpha} f(t) & :=\tilde{\mathcal{I}}_{q, \omega}^{N-\alpha} \tilde{D}_{q, \omega}^{N} f(t) \\
& =\frac{q^{\left(\frac{N}{2}\right)}}{\tilde{\Gamma}_{q}(N-\alpha)} \int_{\omega_{0}}^{t}(\widetilde{t-s})_{q, \omega}^{N-\alpha-1} \tilde{D}_{q, \omega}^{N} f\left(\sigma_{q, \omega}^{N-\alpha-1}(s)\right) \tilde{d}_{q, \omega} s,
\end{aligned}
$$

and ${ }^{C} \tilde{D}_{q, \omega}^{0} f(t)=f(t)$, where $N-1<\alpha<N, N \in \mathbb{N}$.

Theorem 8. For $\alpha, \omega>0,0<q<1$ and $f: I_{q, \omega}^{T} \rightarrow \mathbb{R}$,

$$
{ }^{C} \tilde{D}_{q, \omega}^{\alpha} f(t)=\frac{\left(1-q^{2}\right) q^{\left(\frac{N-\alpha}{2}\right)}}{\tilde{\Gamma}_{q}(N-\alpha)}\left(t-\omega_{0}\right)^{N-\alpha} \sum_{k=0}^{\infty} q^{2 k}\left(1-\widetilde{q^{2 k}+1}\right) \frac{N-\alpha-1}{q, \omega} \tilde{D}_{q, \omega}^{N} f\left(\sigma_{q, \omega}^{2 k+N-\alpha}(s)\right),
$$

where $N-1<\alpha<N, N \in \mathbb{N}$.

Proof. For $t \in I_{q, \omega}^{T}$ and by Definition 5, we have

$$
\begin{aligned}
{ }^{C} \tilde{D}_{q, \omega}^{\alpha} f(t) & =\frac{\left(1-q^{2}\right) q^{\left(\frac{N-\alpha}{2}\right)}}{\tilde{\Gamma}_{q}(N-\alpha)}\left(t-\omega_{0}\right) \sum_{k=0}^{\infty} q^{2 k}\left(t-\widetilde{\sigma_{q, \omega}^{2 k+1}}(t)\right)_{q, \omega}^{\frac{N-\alpha-1}{2}} \tilde{D}_{q, \omega}^{N} f\left(\sigma_{q, \omega}^{2 k+N-\alpha}(s)\right) \\
& =\frac{\left(1-q^{2}\right) q^{\left(\frac{N-\alpha}{2}\right)}}{\tilde{\Gamma}_{q}(N-\alpha)}\left(t-\omega_{0}\right)^{N-\alpha} \sum_{k=0}^{\infty} q^{2 k}\left(1-\widetilde{q^{2 k}+1}\right)_{q, \omega}^{\frac{N-\alpha-1}{D_{q, \omega}}} \tilde{D}_{q,\left(\sigma_{q, \omega}^{2 k+N-\alpha}(s)\right) .}
\end{aligned}
$$

The proof is complete.

Next, we present some properties of fractional symmetric Hahn difference operators of Caputo type as follows.

Theorem 9. For $\alpha, \omega>0,0<q<1$ and $f: I_{q, \omega}^{T} \rightarrow \mathbb{R}$,

$$
{ }^{C} \tilde{D}_{q, \omega}^{\alpha} \tilde{\mathcal{I}}_{q, \omega}^{\alpha} f(t)=f(t) .
$$

Proof. For some $N-1<\alpha<N, N \in \mathbb{N}$ and from Definition 5 and Corollary 1 , we have

$$
\begin{aligned}
{ }^{C} \tilde{D}_{q, \omega}^{\alpha} \tilde{\mathcal{I}}_{q, \omega}^{\alpha} f(t) & =\tilde{\mathcal{I}}_{q, \omega}^{N-\alpha} \tilde{D}_{q, \omega}^{N} \tilde{\mathcal{I}}_{q, \omega}^{\alpha} f(t)=\tilde{\mathcal{I}}_{q, \omega}^{N-\alpha} \tilde{D}_{q, \omega}^{N-\alpha} f(t) \\
& =f(t)-\sum_{k=0}^{N-1} \frac{q^{\left(\begin{array}{c}
{ }_{2} \\
\alpha-\alpha
\end{array}\right)}}{\tilde{\Gamma}_{q}(k-\alpha+1)}\left(t-\omega_{0}\right)^{k-\alpha}\left[\tilde{D}_{q, \omega}^{k} \tilde{\mathcal{I}}_{q, \omega}^{\alpha} f\left(\omega_{0}\right)\right] .
\end{aligned}
$$

From (7), we have

$$
\sum_{k=0}^{N-1} \frac{q^{\left(\frac{k-\alpha}{2}\right)}}{\tilde{\Gamma}_{q}(k-\alpha+1)}\left(t-\omega_{0}\right)^{k-\alpha}\left[\tilde{D}_{q, \omega}^{k} \tilde{\mathcal{I}}_{q, \omega}^{\alpha} f\left(\omega_{0}\right)\right]=0 .
$$

It implies that

$$
{ }^{C} \tilde{D}_{q, \omega}^{\alpha} \tilde{\mathcal{I}}_{q, \omega}^{\alpha} f(t)=f(t) .
$$

The proof is complete. 
Theorem 10. For $\alpha, \omega>0,0<q<1$ and $f: I_{q, \omega}^{T} \rightarrow \mathbb{R}$,

$$
\mathcal{I}_{q, \omega}^{\alpha}{ }^{C} D_{q, \omega}^{\alpha} f(t)=f(t)-\sum_{k=0}^{N-1} \frac{\left(t-\omega_{0}\right)^{k}}{[\tilde{k}]_{q}}\left[D_{q, \omega}^{k} f\left(\omega_{0}\right)\right],
$$

where $N-1<\alpha<N, N \in \mathbb{N}$.

Proof. From Definition 5, Lemma 1a, and Corollary 1, we have

$$
\begin{aligned}
\mathcal{I}_{q, \omega}^{\alpha}{ }^{C} D_{q, \omega}^{\alpha} f(t) & =\mathcal{I}_{q, \omega}^{\alpha}\left[\mathcal{I}_{q, \omega}^{N-\alpha} D_{q, \omega}^{N} f(t)\right]=\mathcal{I}_{q, \omega}^{N} D_{q, \omega}^{N} f(t) \\
& =f(t)-\sum_{k=0}^{N-1} \frac{q^{\left(\frac{k}{2}\right)}}{\tilde{\Gamma}_{q}(k+1)}\left[\tilde{D}_{q, \omega}^{k} f\left(\omega_{0}\right)\right]\left(t-\omega_{0}\right)^{k} \\
& =f(t)-\sum_{k=0}^{N-1} \frac{q^{\left(\frac{k}{2}\right)}}{[\tilde{k}]_{q}}\left[\tilde{D}_{q, \omega}^{k} f\left(\omega_{0}\right)\right]\left(t-\omega_{0}\right)^{k} .
\end{aligned}
$$

The proof is complete.

Corollary 2. Let $\alpha, \omega>0,0<q<1$ and $f: I_{q, \omega}^{T} \rightarrow \mathbb{R}$. Then,

$$
\tilde{\mathcal{I}}_{q, \omega}^{\alpha}{ }^{C} \tilde{D}_{q, \omega}^{\alpha} f(t)=f(t)+C_{0}+C_{1}\left(t-\omega_{0}\right)+\ldots+C_{N-1}\left(t-\omega_{0}\right)^{N-1},
$$

for some $C_{i} \in \mathbb{R}, i=0,1, \ldots, N-1$ and $N-1<\alpha<N, N \in \mathbb{N}$.

\section{Conclusions}

Throughout the paper, fractional symmetric Hahn integral, Riemann-Liouville and Caputo fractional symmetric Hahn difference operators have been introduced. In addition, the properties of these fractional symmetric Hahn operators have been proven. This work might be able to used as a basis for related research, such as defining the Laplace transform for fractional symmetric Hanh calculus or investigating the fractional symmetric Hahn-convolution product and computing its fractional symmetric Hahn-Laplace transform. Finally, we hope to employ these properties to solve symmetric Hahn difference problems in future works.

Author Contributions: Conceptualization, N.P. and T.S.; Methodology, N.P. and T.S.; Validation, N.P. and T.S.; Formal Analysis, N.P. and T.S.; Investigation, N.P. and T.S.; Writing-Original Draft Preparation, N.P. and T.S.; Writing-Review \& Editing, N.P. and T.S.; Funding Acquisition, N.P.

Funding: This research was funded by King Mongkut's University of Technology North Bangkok. Contract no. KMUTNB-61-KNOW-027.

Acknowledgments: The last author of this research was supported by Suan Dusit University.

Conflicts of Interest: The authors declare no conflicts of interest regarding the publication of this paper.

\section{References}

1. Costas-Santos, R.S.; Marcellán, F.; Second structure Relation for $q$-semiclassical polynomials of the Hahn Tableau. J. Math. Anal. Appl. 2007, 329, 206-228. [CrossRef]

2. Kwon, K.H.; Lee, D.W.; Park, S.B.; Yoo, B.H. Hahn class orthogonal polynomials. Kyungpook Math. J. 1998, 38, 259-281.

3. Foupouagnigni, M. Laguerre-Hahn Orthogonal Polynomials with Respect to the Hahn Operator: Fourth-Order Difference Equation for the rth Associated and the Laguerre-Freud Equations Recurrence Coefficients. Ph.D. Thesis, National University of Benin, Proto Novo, Benin, 1998.

4. Hahn, W. Über Orthogonalpolynome, die q-Differenzenlgleichungen genügen. Math. Nachr. 1949, 2, 4-34. [CrossRef] 
5. Aldwoah, K.A. Generalized Time Scales and Associated Difference Equations. Ph.D. Thesis, Cairo University, Cairo, Egypt, 2009.

6. Annaby, M.H.; Hamza, A.E.; Aldwoah, K.A. Hahn difference operator and associated Jackson-Nörlund integrals. J. Optim. Theory Appl. 2012, 154, 133-153. [CrossRef]

7. Malinowska, A.B.; Torres, D.F.M. The Hahn quantum variational calculus. J. Optim. Theory Appl. 2010, 147, 419-442. [CrossRef]

8. Malinowska, A.B.; Torres, D.F.M. Quantum Variational Calculus. In Spinger Briefs in Electrical and Computer Engineering-Control, Automation and Robotics; Springer: Berlin/Heidelberg, Germany, 2014.

9. Malinowska, A.B.; Martins, N. Generalized transversality conditions for the Hahn quantum variational calculus. Optim. J. Math. Program. Oper. Res. 2013, 62, 323-344. [CrossRef]

10. Hamza, A.E.; Ahmed, S.M. Theory of linear Hahn difference equations. J. Adv. Math. 2013, 4, 441-461.

11. Hamza, A.E.; Ahmed, S.M. Existence and uniqueness of solutions of Hahn difference equations. Adv. Differ. Equ. 2013, 2013, 316. [CrossRef]

12. Hamza, A.E.; Makharesh, S.D. Leibniz' rule and Fubinis theorem associated with Hahn difference operator. J. Adv. Math. 2016, 12, 6335-6345.

13. Sitthiwirattham, T. On a nonlocal boundary value problem for nonlinear second-order Hahn difference equation with two different $q, \omega$-derivatives. Adv. Differ. Equ. 2016, 2016, 116. [CrossRef]

14. Sriphanomwan, U.; Tariboon, J.; Patanarapeelert, N.; Ntouyas, S.K.; Sitthiwirattham, T. Nonlocal boundary value problems for second-order nonlinear Hahn integro-difference equations with integral boundary conditions. Adv. Differ. Equ. 2017, 2017, 170. [CrossRef]

15. Brikshavana, T.; Sitthiwirattham, T. On fractional Hahn calculus. Adv. Differ. Equ. 2017, 2017, 354. [CrossRef]

16. Patanarapeelert, N.; Sitthiwirattham, T. Existence Results for Fractional Hahn Difference and Fractional Hahn Integral Boundary Value Problems. Discrete Dyn. Nat. Soc. 2017, 2017, 7895186. [CrossRef]

17. Patanarapeelert, N.; Brikshavana, T.; Sitthiwirattham, T. On nonlocal Dirichlet boundary value problem for sequential Caputo fractional Hahn integrodifference equations. Bound. Value Probl. 2018, 2018, 6. [CrossRef]

18. Patanarapeelert, N.; Sitthiwirattham, T. On Nonlocal Robin Boundary Value Problems for Riemann-Liouville Fractional Hahn Integrodifference Equation. Bound. Value Probl. 2018, 2018, 46. [CrossRef]

19. Dumrongpokaphan, T.; Patanarapeelert, N.; Sitthiwirattham, T. Existence Results of a Coupled System of Caputo Fractional Hahn Difference Equations with Nonlocal Fractional Hahn Integral Boundary Value Conditions. Mathematics 2019, 7, 15. [CrossRef]

20. Artur, M.C.; Cruz, B.; Martins, N.; Torres, D.F.M. Hahn's symmetric quantum variational calculus. Numer. Algebra Control Optim. 2013, 3, 77-94.

21. Sun, M.; Jin, Y.; Hou, C. Certain fractional $q$-symmetric integrals and $q$-symmetric derivatives and their application. Adv. Differ. Equ. 2016, 2016, 222. [CrossRef]

22. Rajkovic P.M.; Marinković S.D. ; Stanković M.S. Fractional integrals and derivatives in $q$-calculus. Appl. Anal. Discrete Math. 2007, 1, 311-323.

(c) 2019 by the authors. Licensee MDPI, Basel, Switzerland. This article is an open access article distributed under the terms and conditions of the Creative Commons Attribution (CC BY) license (http:// creativecommons.org/licenses/by/4.0/). 